\title{
QUASISYMMETRIC EMBEDDINGS OF METRIC SPACES
}

\author{
P. TUKIA and J. VÄISÄLÄ
}

\section{Introduction}

1.1. The theory of quasiconformal maps deals with embeddings $f: G \rightarrow R^{n}$, $G$ open in the euclidean space $R^{n}$. The main motivation for the present paper was the desire to extend the notion of quasiconformality to a more general setting, for example, for embeddings $f: G \rightarrow R^{n}, G$ open in $R^{p}, p<n$. More generally, we consider embeddings $f: X \rightarrow Y$ where $X$ and $Y$ are metric spaces. Using the Polish notation $|a-b|$ for the distance between $a$ and $b$ in any metric space, we are interested in what happens to the ratio

$$
\varrho=\frac{|a-x|}{|b-x|}
$$

for three points $a, b, x$ in $X$ with $b \neq x$. We say that $f$ is quasisymmetric, abbreviated QS, if there is a homeomorphism $\eta:[0, \infty) \rightarrow[0, \infty)$ such that

$$
\frac{|f(a)-f(x)|}{|f(b)-f(x)|} \leqq \eta(\varrho)
$$

$\therefore$ or all such triples $a, b, x$. We also say that $f$ is $\eta$-quasisymmetric if (1.2) is satisfied with a given function $\eta$. Otherwise stated, an embedding $f: X \rightarrow Y$ is $\eta$-QS if and only if $|a-x| \leqq t|b-x|$ implies $|f(a)-f(x)| \leqq \eta(t)|f(b)-f(x)|$ for all $a, b, x \in X$ and $t \geqq 0$. If $f$ is $\eta$-QS with $\eta(t)=t$, we say that $f$ is a similarity. Thus $f$ is a similarity if and only if there is $L>0$ such that $|f(x)-f(y)|=L|x-y|$ for all $x, y \in X$.

A curious fact is that for a large class of spaces $X$, including all connected spaces, every QS embedding $f: X \rightarrow Y$ is $\eta$-QS with $\eta$ of the form $\eta(t)=C \max \left(t^{\alpha}, t^{1 / \alpha}\right)$ (Corollary 3.12).

The term "quasisymmetric" has been used in the theory of quasiconformal maps [LV, II.7.1] for increasing embeddings $f: \Delta \rightarrow R^{1}$, where $\Delta \subset R^{1}$ is an interval, such that for some constant $H$,

$$
|f(a)-f(x)| \leqq H|f(b)-f(x)|
$$

whenever $|a-x| \leqq|b-x|$. The concept was introduced by Beurling and Ahlfors $[\mathrm{BA}]$, the term by Kelingos [Ks]. For general metric spaces, we shall call an embedding 
$f$ weakly quasisymmetric or weakly $H$-quasisymmetric if it satisfies (1.3). Every $\eta$-QS embedding is weakly $H$-QS with $H=\eta(1)$. The converse is not true, except for some important special cases, for example, if $X=R^{p}, Y=R^{n}$. A notion between quasisymmetry and weak quasisymmetry is considered in Theorem 3.10. The weakly QS embeddings in euclidean spaces have been considered by $H$. Renggli $\left[\mathrm{Re}_{1}\right],\left[\mathrm{Re}_{2}\right]$, who called them mappings of bounded triangular dilatation.

In the general case, quasisymmetry seems to be a more natural concept than weak quasisymmetry. For example, the metric spaces and the QS embeddings form a category, but the composition of two weakly QS embeddings need not be weakly QS.

An embedding $f: R^{n} \rightarrow R^{n}$ is quasiconformal if and only if it is QS. If $G$ is open in $R^{n}$, a QS embedding $f: G \rightarrow R^{n}$ is quasiconformal, but the converse is not true. For example, a Möbius transformation of a ball onto a half space is not QS. In fact, a QS image of a bounded space is always bounded. However, the concepts "locally quasisymmetric" and "locally quasiconformal" are equivalent for embeddings and immersions $f: G \rightarrow R^{n}, G$ open in $R^{n}$.

In this paper we consider mainly the properties of quasisymmetry which are independent of any euclidean structure. In particular, we study relations between quasisymmetry and weak quasisymmetry. Section 3 deals with equicontinuity and limits of QS maps. We also prove that a QS embedding of a bounded connected space satisfies a Hölder condition. Finally, in Section 4, we give a characterization of QS arcs. The euclidean case will be considered in a later paper [Vä그.

We wish to thank J. Luukkainen for careful reading of our manuscript and for several valuable suggestions. The results 2.24 and 2.25 are due to him. Originally, we only had a weaker form of 2.25 .

1.4. Notation. All spaces in this paper are metric and usually denoted by $X$ or $Y$. The distance between two points $a, b$ is written as $|a-b|$. We let $d(A, B)$ denote the distance between two sets $A, B$, and $d(A)$ the diameter of a set $A$. The open ball $\left\{x:\left|x-x_{0}\right|<r\right\}$ is written as $B\left(x_{0}, r\right)$ and the closed ball $\left\{x:\left|x-x_{0}\right| \leqq r\right\}$ as $B_{c}\left(x_{0}, r\right) . \mathbf{N}$ is used for the set of positive integers.

\section{Basic concepts}

2.1. Preliminary remarks. In the definition of quasisymmetry, we assumed for the sake of convenience that $\eta:[0, \infty) \rightarrow[0, \infty)$ is a homeomorphism. However, an embedding $f: X \rightarrow Y$ is QS as soon as it satisfies the condition (1.2) with any function $\eta$ which is bounded on bounded sets and has the limit zero at the origin. Indeed, we can then easily find a homeomorphism $\eta_{1}:[0, \infty) \rightarrow[0, \infty)$ such that $\eta(t) \leqq \eta_{1}(t)$ for all $t>0$.

Choosing $a=b$ in (1.2) we see that $\eta(1) \geqq 1$ whenever card $X \geqq 2$. For convenience, we shall assume that $\eta(1) \geqq 1$ also in the trivial case card $X \leqq 1$. Similarly, we shall assume that $H \geqq 1$ in (1.3). 
Changing the roles of $a$ and $b$ in (1.2) we see that an $\eta$-QS embedding satisfies the double inequality

$$
\eta\left(\varrho^{-1}\right)^{-1} \leqq \frac{|f(a)-f(x)|}{|f(b)-f(x)|} \leqq \eta(\varrho)
$$

for all $a, b, x \in X$ with $b \neq x$ and $|a-x| /|b-x|=\varrho$.

The definition of weak quasisymmetry can also be stated as follows: Let $f: X \rightarrow Y$ be an embedding. For $x \in X$ and $r>0$ set

$$
\begin{gathered}
L(x, f, r)=\sup \{|f(y)-f(x)|:|y-x| \leqq r\}, \\
l(x, f, r)=\inf \{|f(y)-f(x)|:|y-x| \geqq r\},
\end{gathered}
$$

with the usual convention inf $\emptyset=\infty$. Then $f$ is weakly $H$-QS if and only if $L(x, f, r) \leqq$ $H l(x, f, r)$ for all $x \in X$ and $r>0$.

The following result is obvious:

2.2. Theorem. If $f: X \rightarrow Y$ is $\eta$-QS, the inverse map $f^{-1}: f X \rightarrow X$ is $\eta^{\prime}$-QS with $\eta^{\prime}(t)=\eta^{-1}\left(t^{-1}\right)^{-1}$ for $t>0$. If $f: X \rightarrow Y$ is $\eta_{1}$-QS and $g: Y \rightarrow Z \quad \eta_{2}$-QS, then gf is $\eta$-QS with $\eta(t)=\eta_{2}\left(\eta_{1}(t)\right)$. A restriction of an $\eta$-QS embedding is $\eta$-QS.

2.3. Examples. If $f$ satisfies a two-sided Lipschitz condition

$$
\left|x-x^{\prime}\right| / L \leqq\left|f(x)-f\left(x^{\prime}\right)\right| \leqq L\left|x-x^{\prime}\right|
$$

for all $x, x^{\prime}$ in $X$, then $f$ is $\eta$-QS with $\eta(t)=L^{2} t$. Conversely, let $f$ be $\eta$-QS with $\eta(t)=\alpha t, \alpha \geqq 1$. Let $x_{0}, x_{1}, x, x^{\prime}$ be points in $X$ with $x \neq x_{1} \neq x_{0}$, and let $y_{0}, y_{1}, y, y^{\prime}$ be their images. Then

$$
\left|y-y^{\prime}\right|=\frac{\left|y-y^{\prime}\right|}{\left|y-y_{1}\right|} \frac{\left|y-y_{1}\right|}{\left|y_{0}-y_{1}\right|}\left|y_{0}-y_{1}\right| \leqq \alpha^{2} \frac{\left|x-x^{\prime}\right|}{\left|x_{0}-x_{1}\right|}\left|y_{0}-y_{1}\right|,
$$

which is easily seen to be true also for $x=x_{1}$. Hence the right-hand side of (2.4) is true with $L=\alpha^{2}\left|y_{0}-y_{1}\right| /\left|x_{0}-x_{1}\right|$. Similarly, the left-hand side is true with $L=\alpha^{2}\left|x_{0}-x_{1}\right| /\left|y_{0}-y_{1}\right|$. However, $L$ cannot be chosen to depend only on $\alpha$, as is seen from the maps $f: R^{n} \rightarrow R^{n}, f(x)=\lambda x, \lambda>0$.

The map $f:[0, \infty) \rightarrow[0, \infty), f(x)=x^{2}$, is $\eta$-QS with $\eta(t)=t^{2}+2 t$. Every quasiconformal map $f: R^{n} \rightarrow R^{n}$ is QS. This follows, for example, from the proof of $\left[V \ddot{a}_{1}, 22.3\right]$ and from Theorem 2.16 of the present paper. Further relations between quasisymmetry and quasiconformality will be considered in [Vä].

In the following example, we consider $R^{2}$ with the metric induced by the norm $|x|=\max \left(\left|x_{1}\right|,\left|x_{2}\right|\right)$. Let $A=\{-1 / 2,0,1 / 2\}, B=\{-1 / 4,0,1 / 2\}, \quad X=\mathbf{N} \times A$ and $Y=\mathbf{N} \times B$. Let $\varphi: A \rightarrow B$ be the unique increasing bijection. Then $f=\operatorname{id} \times \varphi: X \rightarrow Y$ is a homeomorphism, which is $\eta$-QS with $\eta(t)=2 t$. Let $g: Y \rightarrow R^{2}$ be the embedding defined by $g(n,-1 / 4)=(n,-1 / 4 n)$ and by $g(n, a)=(n, a)$ for $a=0,1 / 2$. Then $g$ is weakly 1-QS. However, $g f$ is not weakly QS. On the other hand, $(g f)^{-1}: g Y \rightarrow X$ is weakly QS. Observe that $g$ is not QS. 
2.5. Theorem. Let $f: X \rightarrow Y$ be $\eta$-QS. Let $A \subset B \subset X$ with $d(A)>0, d(B)<\infty$. Then $d(f B)<\infty$ and

$$
1 / 2 \eta\left(\frac{d(B)}{d(A)}\right) \leqq \frac{d(f A)}{d(f B)} \leqq \eta\left(\frac{d(A)}{d(B)}\right) .
$$

Proof. Choose points $b_{n}, b_{n}^{\prime}$ in $B$ such that $\left|b_{n}^{\prime}-b_{n}\right| \geqq d(B) / 2$ and that $\left|b_{n}^{\prime}-b_{n}\right| \rightarrow d(B)$ as $n \rightarrow \infty$. If $b \in B,\left|b-b_{1}\right| \leqq 2\left|b_{1}^{\prime}-b_{1}\right|$, which implies $\left|f(b)-f\left(b_{1}\right)\right| \leqq \eta(2)\left|f\left(b_{1}^{\prime}\right)-f\left(b_{1}\right)\right|$. Hence $d(f B) \leqq 2 \eta(2)\left|f\left(b_{1}^{\prime}\right)-f\left(b_{1}\right)\right|<\infty$.

Let $a \in A$ and $n \in \mathbf{N}$. Then either $2\left|a-b_{n}\right| \geqq\left|b_{n}^{\prime}-b_{n}\right|$ or $2\left|a-b_{n}^{\prime}\right| \geqq\left|b_{n}^{\prime}-b_{n}\right|$, say $2\left|a-b_{n}\right| \geqq\left|b_{n}^{\prime}-b_{n}\right|$. If $\quad x \in A$, then $|f(x)-f(a)| \leqq \eta\left(|x-a| /\left|b_{n}-a\right|\right)\left|f\left(b_{n}\right)-f(a)\right| \leqq$ $\eta\left(2 d(A) /\left|b_{n}^{\prime}-b_{n}\right|\right) d(f B)$. Hence $d(f A) \leqq \eta\left(2 d(A) /\left|b_{n}^{\prime}-b_{n}\right|\right) d(f B)$, which yields the second inequality as $n \rightarrow \infty$.

Since $f^{-1}: f X \rightarrow X$ is $\eta^{\prime}$-QS with $\eta^{\prime}(t)=\eta^{-1}\left(t^{-1}\right)^{-1}$, the first inequality follows from the second one.

2.6. Corollary. A QS embedding maps every bounded set onto a bounded set.

2.7. Definitions. A space $X$ is pseudoconvex if there is an increasing function $C:[1, \infty) \rightarrow[1, \infty)$ with the following property: If $a, b \in X$ and $0<r \leqq|a-b|$, then there is a finite sequence of points $a=a_{0}, a_{1}, \ldots, a_{s}=b$ such that $s \leqq C(|a-b| / r)$ and $\left|a_{j+1}-a_{j}\right| \leqq\left|a_{j}-a_{j-1}\right| \leqq r$ for $j=1, \ldots, s-1$. We also say that $X$ is $C$-pseudoconvex if this is true with a given function $C$.

A space $X$ is homogeneously totally bounded, abbreviated HTB, if there is an increasing function $k:[1 / 2, \infty) \rightarrow[1, \infty)$ such that for every $\alpha \geqq 1 / 2$, every closed ball $B_{c}(x, r)$ can be covered with sets $A_{1}, \ldots, A_{s}$ such that $s \leqq k(\alpha)$ and $d\left(A_{j}\right)<r / \alpha$ for all $j$. We also say that $X$ is $k$-HTB.

A space $X$ is of bounded turning, abbreviated BT, if there is $c \geqq 1$ such that each pair of distinct points $a, b$ in $X$ can be joined by an $\operatorname{arc} A$ with $d(A) \leqq c|a-b|$. We also say that $X$ is $c$-BT.

2.8. Remarks. Every convex set in a normed vector space is 1-BT and $C$-pseudoconvex with $C(\alpha)=\alpha+1$. Every subset of a $k$-HTB space is $k$-HTB. Every subset of $R^{n}$ is $k$-HTB with $k(\alpha)=2^{n}\left(\alpha n^{1 / 2}+1\right)^{n}$. Every HTB space is separable. The term "bounded turning" has been used for arcs and topological circles in the theory of quasiconformal maps [LV, II.8.7 and II.8.8]. The set $X=\left\{(x, y) \in R^{2}: x \geqq 0,|y|=x^{2}\right\}$ is neither pseudoconvex nor BT. The set of rational numbers is pseudoconvex but not BT.

If $X$ is $k$-HTB and if $a_{1}, \ldots, a_{s}$ are points in $B_{c}(x, r)$ with $\left|a_{i}-a_{j}\right| \geqq t>0$ whenever $i \neq j$, then $s \leqq k(r / t)$.

2.9. Theorem. If $X$ is $k$-HTB and $c$-BT, then $X$ is $C$-pseudoconvex with $C(\alpha)=k(c \alpha)$. 
Proof. Let $a, b \in X$ and let $0<r<|a-b|$. Choose an $\operatorname{arc} A \subset X$ joining $a$ and $b$ such that $d(A) \leqq c|a-b|$. Let $\leqq$ be the natural ordering of $A$ with $a<b$. Let $a_{0}=a$ and define inductively points $a_{j}=\max \left\{x \in A:\left|x-a_{j-1}\right| \leqq r\right\}$. Then $\left|a_{i}-a_{j}\right| \geqq r$ if $i<j$ and $a_{j} \neq b$. Hence $a_{s}=b$ for some $s$. Since $A \subset B_{c}(a, c|a-b|)$, we have $s \leqq k(c|a-b| / r)$ for the first such $s$.

2.10. Theorem. If $X$ is $k$-HTB and if $f: X \rightarrow Y$ is $\eta$-QS, then $f X$ is $k^{\prime}$-HTB, where $k^{\prime}$ depends only on $k$ and $\eta$.

Proof. We may assume that $f X=Y$. Let $y \in Y, r>0$ and $\alpha \geqq 1 / 2$. Set $x=f^{-1}(y)$ and $L=\sup \left|f^{-1}(z)-f^{-1}(y)\right|$ over $z \in B_{c}(y, r)$. We may assume $L>0$. Set $\beta=2 / \eta^{-1}(1 / 2 \alpha)$. Since $X$ is $k$-HTB, there is a covering $A_{1}, \ldots, A_{s}$ of $B_{c}(x, L)$ such that $s \leqq k(\beta)$ and $d\left(A_{j}\right)<L / \beta$ for all $j$. Set $B=f^{-1} B_{c}(y, r)$ and $A_{j}^{\prime}=A_{j} \cap B$. Then the sets $f A_{j}^{\prime}$ cover $B_{c}(y, r)$. Observing that $d(B) \geqq L$ and applying 2.5 with $A=A_{j}^{\prime}$, we obtain

$$
d\left(f A_{j}^{\prime}\right) \leqq \eta\left(\frac{2 d\left(A_{j}^{\prime}\right)}{d(B)}\right) d\left(B_{c}(y, r)\right) \leqq \eta(2 / \beta) \cdot 2 r=r / \alpha .
$$

Hence $Y$ is $k^{\prime}$-HTB with $k^{\prime}(\alpha)=k\left(2 / \eta^{-1}(1 / 2 \alpha)\right)$.

2.11. Theorem. Let $X$ be $c$-BT and let $f: X \rightarrow Y$ be $\eta$-QS. Then $f X$ is $2 \eta(c)$-BT.

Proof. Let $a, b \in X, a \neq b$. Choose an arc $A$ in $X$ joining $a$ and $b$ such that $d(A) \leqq c|a-b|$. If $x \in A$, then $|x-a| \leqq c|a-b|$, and therefore $|f(x)-f(a)| \leqq$ $\eta(c)|f(a)-f(b)|$. Consequently, $d(f A) \leqq 2 \eta(c)|f(a)-f(b)|$.

2.12. Lemma. Suppose that $f: X \rightarrow Y$ is weakly $H$-QS and that $X$ is $C$-pseudoconvex. If $a, b, x \in X$ and if $|a-x| /|b-x|=\varrho \geqq 1$, then $|f(a)-f(x)| \leqq \eta(\varrho)|f(b)-f(x)|$ where $\eta(\varrho)=C(\varrho) H^{C(\varrho)}$.

Proof. Set $|b-x|=r$. By pseudoconvexity, there are points $x=x_{0}, x_{1}, \ldots, x_{s}=a$ such that $\left|x_{j+1}-x_{j}\right| \leqq\left|x_{j}-x_{j-1}\right| \leqq r$ and $s \leqq C(\varrho)$. Then $\left|f\left(x_{1}\right)-f(x)\right| \leqq$ $H|f(x)-f(b)|,\left|f\left(x_{2}\right)-f\left(x_{1}\right)\right| \leqq H^{2}|f(x)-f(b)|$ and so on. This yields $|f(a)-f(x)| \leqq$ $\sum_{j}\left|f\left(x_{j}\right)-f\left(x_{j-1}\right)\right| \leqq \sum_{j} H^{j}|f(x)-f(b)| \leqq s H^{s}|f(x)-f(b)|$.

2.13. Remark. Lemma 2.12 does not say that $f$ is QS, since $\eta(\varrho)$ is not defined for $\varrho<1$. In fact, $f$ need not be QS; see the map $f$ in the last paragraph of this paper.

The following result follows directly from the definition of pseudoconvexity:

2.14. Lemma. Suppose that $X$ is pseudoconvex, that $a, b$ are distinct points in $X$ and that $0 \leqq k<k^{\prime} \leqq 1$. Then there is $x \in X$ such that $k|b-a|<|x-a|<$ $k^{\prime}|b-a|$.

2.15. Theorem. Suppose that $X$ is $C$-pseudoconvex and that $Y$ is $k$-HTB. Then every weakly $H$-QS embedding $f: X \rightarrow Y$ is $\eta$-QS, where $\eta$ depends only on $C, k$ and $H$. 
Proof. Let $a, b, x$ be distinct points in $X$ with $|a-x| /|b-x|=\varrho$, and set $\varrho^{\prime}=$ $|f(a)-f(x)| /|f(b)-f(x)|$. By $2.12, \varrho^{\prime} \leqq C(\varrho) H^{C(\varrho)}$ if $\varrho \geqq 1$. For $\varrho<1$ we know that $\varrho^{\prime} \leqq H$, but we need an estimate $\varrho^{\prime} \leqq \eta(\varrho)$ where $\eta(\varrho) \rightarrow 0$ as $\varrho \rightarrow 0$.

Suppose that $\varrho<1$. By 2.14, we can find a sequence $b_{0}, b_{1}, \ldots$ of points in $X$ such that $b_{0}=b$ and such that $\left|b_{j-1}-x\right| / 4<\left|b_{j}-x\right|<\left|b_{j-1}-x\right| / 3$ for all $j \geqq 1$. Then $b_{j} \rightarrow x$. Let $s$ be the least integer $j$ such that $\left|b_{j}-x\right|<|a-x|$. Since $\left|b_{j}-x\right|>$ $4^{-j}|b-x|$, we obtain $\varrho>4^{-s}$.

Suppose that $0 \leqq i<j<s$. Then $\left|x-b_{j}\right| \leqq\left|x-b_{i+1}\right|<\left|x-b_{i}\right| / 3 \leqq\left|x-b_{j}\right| / 3$ $+\left|b_{i}-b_{j}\right| / 3$, which implies $2\left|x-b_{j}\right| \leqq\left|b_{i}-b_{j}\right|$. Since $|a-x| \leqq\left|b_{j}-x\right|$, this yields $\left|a-b_{j}\right| \leqq\left|b_{i}-b_{j}\right|$. Thus $2 H\left|f\left(b_{i}\right)-f\left(b_{j}\right)\right| \geqq\left|f(a)-f\left(b_{j}\right)\right|+\left|f(x)-f\left(b_{j}\right)\right| \geqq|f(a)-f(x)|$. Hence the distances between the points $f\left(b_{j}\right), 0 \leqq j \leqq s-1$, are at least $|f(a)-f(x)| / 2 H$. On the other hand, $\left|b_{j}-x\right| \leqq|b-x|$ implies $\left|f\left(b_{j}\right)-f(x)\right| \leqq H|f(b)-f(x)|$. Since $Y$ is $k$-HTB, this yields $s \leqq k\left(2 H^{2} / \varrho^{\prime}\right)$. Since $\varrho>4^{-s}$, we obtain

$$
\log \frac{1}{\varrho}<k\left(2 H^{2} / \varrho^{\prime}\right) \log 4 .
$$

Since the right side is decreasing in $\varrho^{\prime}$, we obtain $\varrho^{\prime} \leqq \eta(\varrho)$ with $\eta(\varrho) \rightarrow 0$ as $\varrho \rightarrow 0$.

2.16. Theorem. Let $X \subset R^{p}$ be $c$-BT, and let $f: X \rightarrow R^{n}$ be weakly $H-\mathrm{QS}$. Then $f$ is $\eta-\mathrm{QS}$, and $f X$ is $c^{\prime}$ - $\mathrm{BT}$, where $\eta$ and $c^{\prime}$ depend only on $p, n, c$ and $H$.

Proof. Since euclidean spaces are HTB, this follows directly from 2.9, 2.11 and 2.15 .

2.17. Remark. It follows from 2.16 that the subsets of euclidean spaces which are of bounded turning form a convenient class of spaces in the theory of QS maps, since (1) it is closed with respect to QS embeddings and (2) there is no difference between quasisymmetry and weak quasisymmetry. In particular, for maps of an interval $\Delta \subset R^{1}$ into $R^{1}$, our definition of quasisymmetry is equivalent to the usual one, except that one customarily only considers increasing maps.

2.18. Continuity. Suppose that $f: X \rightarrow Y$ is a function (not necessarily continuous) and that there is $H \geqq 1$ such that

$$
|f(a)-f(x)| \leqq H|f(b)-f(x)|
$$

whenever $|a-x| \leqq|b-x|$. Thus, if $f$ is an embedding, $f$ is weakly $H$-QS. In general, a function satisfying (2.19) need not be continuous. For example, let $X$ be any space and let $Y$ be $X$ with the metric $|a-b|=1$ for $a \neq b$. Then the identity map $f: X \rightarrow Y$ satisfies (2.19) with $H=1$, but it is not usually continuous. Furthermore, even if $f$ is continuous, it need not be an embedding, since a constant function always satisfies (2.19). We give two results which show that in certain important cases, conditions like this imply that $f$ is an embedding. 
2.20. Theorem. Suppose that $H \geqq 1$ and that $f: X \rightarrow Y$ is a function satisfying (2.19) whenever $|a-x| \leqq|b-x|$. If $f$ is not constant in any non-empty open set, then $f$ is injective and $f^{-1}: f X \rightarrow X$ is continuous. If $X$ is connected, this is true whenever $f$ is non-constant. If every bounded set in $Y$ is totally bounded, $f$ is continuous.

Proof. Suppose that $f$ is not constant in any non-empty open set. If $f(x)=f(y)$ for some $x \neq y$, then $|f(x)-f(z)| \leqq H|f(x)-f(y)|=0$ for all $z$ in $B(x,|x-y|)$, which is impossible. Hence $f$ is injective. Let $x_{0} \in X$ and $\varepsilon>0$. Since $\left\{x_{0}\right\}$ is not open in $X$, there is $x_{1} \in B\left(x_{0}, \varepsilon\right), x_{1} \neq x_{0}$. If $\left|x-x_{0}\right| \geqq \varepsilon$, then $\left|f(x)-f\left(x_{0}\right)\right| \geqq\left|f\left(x_{1}\right)-f\left(x_{0}\right)\right| / H=$ $\delta>0$. Hence $f B\left(x_{0}, \varepsilon\right)$ contains $B\left(f\left(x_{0}\right), \delta\right) \cap f X$, which implies that $f^{-1}$ is continuous.

Next assume that $X$ is connected and that $f$ is not injective. By what was proved above, there is $y \in Y$ such that int $f^{-1}(y)=U \neq \emptyset$. If $f$ is not constant, $U$ has a boundary point $x$. Choose $b$ and $c$ in $U$ such that $|x-c| \leqq|b-c|$. Then $|f(x)-y| \leqq H|y-y|=0$. Hence, if $|a-x|<|b-x|,|f(a)-y| \leqq H|f(b)-y|=0$. Thus $B(x,|b-x|) \subset U$, which is a contradiction.

Finally, assume that all bounded sets in $Y$ are totally bounded. If $f$ is not continuous at $x_{0} \in X$, there is a sequence of points $x_{j}$ converging to $x_{0}$ such that (1) $\left|x_{j}-x_{0}\right| \leqq\left|x_{1}-x_{0}\right|$, (2) $\left|x_{j}-x_{0}\right| \leqq\left|x_{j}-x_{i}\right|$ for $i<j$, (3) $\left|f\left(x_{j}\right)-f\left(x_{0}\right)\right| \geqq r>0$. Since $\left|f\left(x_{j}\right)-f\left(x_{0}\right)\right| \leqq H\left|f\left(x_{1}\right)-f\left(x_{0}\right)\right|$, the set of all points $f\left(x_{j}\right)$ is totally bounded. Consequently, there are arbitrarily small distances $\left|f\left(x_{i}\right)-f\left(x_{j}\right)\right|, i \neq j$. On the other hand, $i<j$ implies $\left|f\left(x_{i}\right)-f\left(x_{j}\right)\right| \geqq\left|f\left(x_{j}\right)-f\left(x_{0}\right)\right| / H \geqq r / H$. This contradiction proves that $f$ is continuous.

2.21. Theorem. Let $\eta:[0, \infty) \rightarrow[0, \infty)$ be a homeomorphism. Let $f: X \rightarrow Y$ be a function such that

$$
|f(a)-f(x)| \leqq \eta(\varrho)|f(b)-f(x)|
$$

whenever $|a-x| \leqq \varrho|b-x|$. Then $f$ is either constant or an $\eta$-QS embedding.

Proof. Let $x_{0} \in X$ and $\varepsilon>0$. Fix $b \in X, b \neq x_{0}$. Choose $\varrho>0$ such that $\eta(\varrho)\left|f(b)-f\left(x_{0}\right)\right|<\varepsilon$. Then $\left|f(x)-f\left(x_{0}\right)\right|<\varepsilon$ for $x \in B\left(x_{0}, \varrho\left|b-x_{0}\right|\right)$. Thus $f$ is continuous. Suppose that $f$ is not constant. If $f(x)=f(y)$ for some $x \neq y$ and if $z \neq x$, then, setting $\varrho=|z-x| /|y-x|$, we obtain $|f(z)-f(x)| \leqq \eta(\varrho)|f(y)-f(x)|=0$. Hence $f$ is injective. It remains to prove that $f^{-1}: f X \rightarrow X$ is continuous at an arbitrary point $f\left(x_{0}\right)$. If $x_{0}$ is not isolated in $X$, this can be proved as in 2.20. Suppose that $x_{0}$ is isolated in $X$. We must show that $f\left(x_{0}\right)$ is isolated in $f X$. If this is not true, there is a sequence of points $x_{j} \in X, x_{j} \neq x_{0}$, such that $f\left(x_{j}\right) \rightarrow f\left(x_{0}\right)$. Choose $\varrho>0$ such that $\left|x_{1}-x_{0}\right| \leqq \varrho\left|x_{j}-x_{0}\right|$ for all $j \geqq 1$. Then $\left|f\left(x_{1}\right)-f\left(x_{0}\right)\right| \leqq \eta(\varrho)\left|f\left(x_{j}\right)-f\left(x_{0}\right)\right| \rightarrow 0$, which gives a contradiction.

2.22. Local quasisymmetry. The concepts locally quasisymmetric, locally $\eta$-quasisymmetric, locally weakly quasisymmetric, and locally weakly $H$-quasisymmetric are defined in the obvious way. For example, an embedding $f: X \rightarrow Y$ is locally $\eta$-QS 
if every point $x$ in $X$ has a neighborhood $U$ such that $f \mid U$ is $\eta$-QS. These concepts are also meaningful for immersions.

2.23. Theorem. Suppose that $X$ is compact. If an embedding $f: X \rightarrow Y$ is locally $\mathrm{QS}$ or locally weakly $\mathrm{QS}$, then $f$ is $\mathrm{QS}$ or weakly $\mathrm{QS}$, respectively.

Proof. We prove the QS case; the weakly QS case is easier. Let $f: X \rightarrow Y$ be a locally QS embedding. Choose an open covering $\left\{U_{1}, \ldots, U_{k}\right\}$ of $X$ and a function $\eta$ such that every $f \mid U_{j}$ is $\eta$-QS. Let $\lambda>0$ be a Lebesgue number for the covering $\left(U_{j}\right)$ such that $\lambda<d\left(U_{j}\right)$ whenever $d\left(U_{j}\right)>0$. By compactness, there is $\delta>0$ such that $\left|x-x^{\prime}\right| \geqq \lambda / 2$ implies $\left|f(x)-f\left(x^{\prime}\right)\right| \geqq \delta$. Let $a, b, x$ be distinct points in $X$, and set $\varrho=|a-x| /|b-x|, \varrho^{\prime}=|f(a)-f(x)| /|f(b)-f(x)|$. We divide the rest of the proof into four cases.

Case 1. $|a-x| \leqq \lambda / 2$ and $|b-x| \leqq \lambda / 2$. Then there is $j$ such that $\{a, b, x\} \subset U_{j}$, and thus $\varrho^{\prime} \leqq \eta(\varrho)$.

Case 2. $|a-x| \leqq \lambda / 2$ and $|b-x|>\lambda / 2$. Then there is $j$ such that $\{a, x\} \subset U_{j}$. Since $\lambda<d\left(U_{j}\right)$, there is $y \in U_{j}$ such that $2|y-x| \geqq \lambda$. Then $|a-x| /|y-x| \leqq 2 \varrho d(X) / \lambda$, and we obtain

$$
\varrho^{\prime}=\frac{|f(a)-f(x)|}{|f(y)-f(x)|} \frac{|f(y)-f(x)|}{|f(b)-f(x)|} \leqq \eta\left(\frac{|a-x|}{|y-x|}\right) d(f X) / \delta \leqq \eta(2 \varrho d(X) / \lambda) d(f X) / \delta .
$$

Case 3. $|a-x|>\lambda / 2$ and $|b-x| \leqq \lambda / 2$. A modification of the proof of Case 2 yields the same estimate for $\varrho^{\prime}$.

Case 4. $|a-x|>\lambda / 2$ and $|b-x|>\lambda / 2$. Then $\varrho \geqq \lambda / 2 d(X)$ and $\varrho^{\prime} \leqq d(f X) / \delta$.

As mentioned in the introduction, Theorems 2.24 and 2.25 are due to J. Luukkainen. See also [ $\mathrm{Re}_{1}$, Theorem 4].

2.24. Theorem. Let $f: X \rightarrow Y$ be $\eta$-QS. Then $f$ maps every Cauchy sequence in $X$ to a Cauchy sequence. If $X$ is totally bounded or complete, then $f X$ is totally bounded or complete, respectively.

Proof. We may assume that $f X=Y$. Let $\left(x_{j}\right)$ be a Cauchy sequence in $X$. Then $B=\left\{x_{j}: j \in \mathbf{N}\right\}$ is bounded, and Theorem 2.5 implies

$$
\left|f\left(x_{i}\right)-f\left(x_{j}\right)\right| \leqq \eta\left(\frac{2\left|x_{i}-x_{j}\right|}{d(B)}\right) d(f B) .
$$

Hence the sequence $\left(f\left(x_{j}\right)\right)$ is Cauchy.

A space is totally bounded if and only if every sequence has a Cauchy subsequence. Consequently, if $X$ is totally bounded, so is $Y$.

Suppose that $X$ is complete. Let $\left(y_{j}\right)$ be a Cauchy sequence in $Y$. Since $f^{-1}$ is QS, it follows from the first part of the theorem that the sequence $\left(f^{-1}\left(y_{j}\right)\right)$ is Cauchy and hence converges to a point $x \in X$. Thus $\left(y_{j}\right)$ converges to $f(x)$. 
2.25. Theorem. Suppose that $X$ and $Y$ are spaces, that $A \subset X$, that $f: A \rightarrow Y$ is $\eta$-QS and that $\overline{f A}$ is complete. Then f can be extended to an $\eta$-QS embedding $g: \bar{A} \rightarrow Y$.

Proof. By 2.24, the image of every Cauchy sequence in $A$ is convergent. Hence $f$ has a unique extension to a continuous map $g: \bar{A} \rightarrow Y$. Since $\eta$ is continuous, $g$ satisfies the inequality $|g(a)-g(x)| \leqq \eta(\varrho)|g(b)-g(x)|$ whenever $|a-x| \leqq \varrho|b-x|$. By $2.21, g$ is $\eta$-QS.

\section{Equicontinuity and Hölder continuity}

3.1. We recall that a family $F$ of maps $f: X \rightarrow Y$ is equicontinuous if for every $x_{0} \in X$ and $\varepsilon>0$ there is a neighborhood $U$ of $x_{0}$ such that $\left|f(x)-f\left(x_{0}\right)\right|<\varepsilon$ whenever $x \in U$ and $f \in F$.

3.2. Theorem. Let $H \geqq 1$ and let $X$ and $Y$ be spaces with $Y$ totally bounded. Then the family $F$ of all weakly $H$-QS embeddings $f: X \rightarrow Y$ is equicontinuous.

Proof. Let $x_{0} \in X$ and let $\varepsilon>0$. We may assume that $x_{0}$ is not isolated in $X$. Choose an integer $k$ such that $Y$ can be covered with $k$ sets of diameter less than $\varepsilon / 2 H$. Choose a sequence of distinct points $x_{j}$ converging to $x_{0}$ such that $\left|x_{j}-x_{0}\right| \leqq$ $\min \left(\left|x_{i}-x_{0}\right|,\left|x_{j}-x_{i}\right| / 2\right)$ for $i<j$. Set $\delta=\left|x_{k+1}-x_{0}\right|$. We shall show that $\left|f(x)-f\left(x_{0}\right)\right|<\varepsilon$ whenever $f \in F$ and $\left|x-x_{0}\right|<\delta$. Choose integers $i<j \leqq k+1$ such that $\left|f\left(x_{i}\right)-f\left(x_{j}\right)\right|<\varepsilon / 2 H$. Since $\left|x_{j}-x_{0}\right| \leqq\left|x_{j}-x_{i}\right| / 2 \leqq\left|x_{j}-x_{i}\right|, \quad\left|f\left(x_{j}\right)-f\left(x_{0}\right)\right| \leqq$ $H\left|f\left(x_{j}\right)-f\left(x_{i}\right)\right|<\varepsilon / 2$. Since $\left|x_{j}-x\right| \leqq\left|x_{j}-x_{0}\right|+\left|x_{0}-x\right|<\left|x_{j}-x_{0}\right|+\delta \leqq 2\left|x_{j}-x_{0}\right| \leqq$ $\left|x_{j}-x_{i}\right|,\left|f\left(x_{j}\right)-f(x)\right| \leqq H\left|f\left(x_{j}\right)-f\left(x_{i}\right)\right|<\varepsilon / 2$. Hence $\left|f(x)-f\left(x_{0}\right)\right|<\varepsilon$.

3.3. Remark. Theorem 3.2 cannot be extended to the case where $Y$ is not totally bounded, even if weak quasisymmetry is replaced by quasisymmetry. For example, the maps $f: R^{n} \rightarrow R^{n}, f(x)=\lambda x, \lambda>0$, are similarities, but their family is not equicontinuous. However, we obtain equicontinuity by either putting an additional condition on the maps or by assuming that the spaces are bounded.

3.4. Theorem. Let $\eta:[0, \infty) \rightarrow[0, \infty)$ be a homeomorphism. Let $X$ and $Y$ be spaces, let $M>0$, and let $a, b$ be distinct points in $X$. Then the family $F$ of all $\eta$-quasisymmetric embeddings $f: X \rightarrow Y$ such that $|f(a)-f(b)| \leqq M$ is equicontinuous.

Proof. Let $x_{0} \in X, x_{0} \neq a$. Then

$$
\left|f(x)-f\left(x_{0}\right)\right| \leqq \eta\left(\frac{\left|x-x_{0}\right|}{\left|a-x_{0}\right|}\right)\left|f(a)-f\left(x_{0}\right)\right| \leqq \eta\left(\frac{\left|x-x_{0}\right|}{|a-b|}\right) \eta\left(\frac{\left|a-x_{0}\right|}{|a-b|}\right) M .
$$

Hence $F$ is equicontinuous at $x_{0}$. For $x_{0}=a$ change the roles of $a$ and $b$.

3.5. Theorem. Let $X$ and $Y$ be bounded spaces and let $\eta:[0, \infty) \rightarrow[0, \infty)$ be a homeomorphism. Then the family $F$ of all $\eta$-QS embeddings $f: X \rightarrow Y$ is uniformly 
equicontinuous. More precisely, if $x, y \in X, x \neq y, f \in F$, then

$$
d(f X)) / 2 \eta\left(\frac{d(X)}{|x-y|}\right) \leqq|f(x)-f(y)| \leqq \eta\left(\frac{2|x-y|}{d(X)}\right) d(f X) \leqq \eta\left(\frac{2|x-y|}{d(X)}\right) d(Y) .
$$

Proof. This is a special case of 2.5 .

3.6. Remarks. Let $F$ be an equicontinuous family of maps $f: X \rightarrow Y$, and let $\bar{F}$ be the closure of $F$ in the compact-open topology of the space $C(X, Y)$ of all continuous maps $f: X \rightarrow Y$. From the Ascoli theorem [Du, 12.6.4] it follows that $\bar{F}$ is compact if the set $F[x]=\{f(x): f \in F\}$ has a compact closure for every $x \in X$. In particular, this is true whenever $Y$ is compact. In the situation of 3.2, the completion $Z$ of $Y$ is compact. Thus $F$ has a compact closure in $C(X, Z)$. In the situation of $3.4, \bar{F}$ is compact if we assume, in addition, that $Y$ is boundedly compact and that $F\left[x_{0}\right]$ is bounded for some $x_{0} \in X$. Indeed, the proof of 3.4 shows that $F[x]$ is then bounded for every $x \in X$.

It follows from 2.20 and 2.21 that the family $\vec{F} \backslash F$ in $3.2,3.4$ and in 3.5 is fairly small. For example, we easily obtain the following result:

3.7. Theorem. Let $f_{j}: X \rightarrow Y$ be a sequence of $\eta$-QS embeddings converging pointwise to a function $f: X \rightarrow Y$. Then $f$ is either constant or $\eta$-QS. Moreover, the convergence is uniform on every compact set.

Proof. The first assertion follows from 2.21. We may assume that $X$ contains two distinct points $a$ and $b$. Since $\left(f_{j}\right)$ is convergent, the set of all distances $\left|f_{j}(a)-f_{j}(b)\right|$ is bounded. By Theorem 3.4 , the family $\left\{f_{j}: j \in \mathbf{N}\right\}$ is equicontinuous. The second assertion then follows from [Ky, 7.15, p. 232].

3.8. HD-spaces. Our next goal is to show that with some mild restrictions on $X$, every QS embedding $f: X \rightarrow Y$ is Hölder continuous and $\eta$-QS with $\eta$ of the form $\eta(t)=C \max \left(t^{\alpha}, t^{1 / \alpha}\right)$. For this purpose, we introduce the following concept: A space $X$ is said to be homogeneously dense, abbreviated HD, if there are numbers $\lambda_{1}, \lambda_{2}$ such that $0<\lambda_{1} \leqq \lambda_{2}<1$ and such that for each pair of points $a, b$ in $X$ there is $x \in X$ satisfying the condition $\lambda_{1}|b-a| \leqq|x-a| \leqq \lambda_{2}|b-a|$. We also say that $X$ is $\left(\lambda_{1}, \lambda_{2}\right)$-HD or simply $\lambda$-HD, $\lambda=\left(\lambda_{1}, \lambda_{2}\right)$.

A connected space is $\lambda$-HD for every $\lambda \in(0,1)^{2}$ with $\lambda_{1} \leqq \lambda_{2}$. A pseudoconvex space is $\lambda$-HD for every $\lambda \in(0,1)^{2}$ with $\lambda_{1}<\lambda_{2}$. The space $[0,1] \cup[2,3]$ and the Cantor middle-third set are HD but neither connected nor pseudoconvex. A HD space containing at least two points has no isolated points.

3.9. Lemma. (1) Let $X$ be $\left(\lambda_{1}, \lambda_{2}\right)$-HD and let $n$ be a positive integer. Then $X$ is $\left(\lambda_{1}^{n}, \lambda_{2}^{n}\right)-\mathrm{HD}$.

(2) Let $X$ be $\lambda$-HD and let $f: X \rightarrow Y$ be $\eta$-QS. Then $f X$ is $\mu$-HD, where $\mu$ depends only on $\eta$ and $\lambda$. 
Proof. Iteration of the definition of the property HD yields (1). To prove (2), choose an integer $n=n(\eta, \lambda)$ such that $\eta\left(\lambda_{2}^{n}\right)<1$. Let $a, b \in X$. By (1), there is $x \in X$ such that $\lambda_{1}^{n}|b-a| \leqq|x-a| \leqq \lambda_{2}^{n}|b-a|$. Hence

$$
\eta\left(\lambda_{1}^{-n}\right)^{-1}|f(b)-f(a)| \leqq|f(x)-f(a)| \leqq \eta\left(\lambda_{2}^{n}\right)|f(b)-f(a)|,
$$

which proves (2).

3.10. Theorem. Suppose that $0<\lambda_{1} \leqq \lambda_{2}<1$, that $1 / \lambda_{1} \leqq h \leqq H$ and that $X$ is $\lambda$-HD. Suppose also that $f: X \rightarrow Y$ is an embedding such that

(1) $|a-x| \leqq h|b-x|$ implies $|f(a)-f(x)| \leqq H|f(b)-f(x)|$,

(2) $|a-x| \leqq|b-x| / H$ implies $|f(a)-f(x)| \leqq|f(b)-f(x)| / h$.

Then there are $C \geqq 1$ and $\alpha \geqq 1$, depending only on $\lambda, h$ and $H$, such that $f$ is $\eta$-QS with $\eta(t)=C \max \left(t^{\alpha}, t^{1 / \alpha}\right)$.

Proof. Let $a, b, x$ be distinct points in $X$, and set $\varrho=|a-x| /|b-x|, \varrho^{\prime}=$ $|f(a)-f(x)| /|f(b)-f(x)|$. We must find $C$ and $\alpha$ such that

$$
\varrho^{\prime} \leqq C \max \left(\varrho^{\alpha}, \varrho^{1 / \alpha}\right) .
$$

Case 1. $\varrho \geqq 1$. Since $X$ is $\lambda$-HD, there is a finite sequence of points $a=x_{0}, x_{1}, \ldots, x_{s}, s \geqq 0$, such that

$$
\lambda_{1}\left|x_{i}-x\right| \leqq\left|x_{i+1}-x\right| \leqq \lambda_{2}\left|x_{i}-x\right|, \quad \lambda_{1}\left|x_{s}-x\right| \leqq|b-x| \leqq\left|x_{s}-x\right| .
$$

Set $x_{s+1}=b$. Since $h \geqq 1 / \lambda_{1},\left|x_{i}-x\right| \leqq h\left|x_{i+1}-x\right|$ for all $i \in[0, s]$, and therefore $\left|f\left(x_{i}\right)-f(x)\right| \leqq H\left|f\left(x_{i+1}\right)-f(x)\right|$. Hence $|f(a)-f(x)| \leqq H^{s+1}|f(b)-f(x)|$, which gives $\varrho^{\prime} \leqq H^{s+1}$. On the other hand, $|b-x| \leqq\left|x_{s}-x\right| \leqq \lambda_{2}^{s}|a-x|$, which yields $\lambda_{z}^{-s} \leqq \varrho$ and thus $H^{s} \leqq \varrho^{\alpha}$ with $\alpha=-\log H / \log \lambda_{2}$. Consequently, $\varrho^{\prime} \leqq H \varrho^{\alpha}$.

Case 2. $\varrho<1$. Choose an integer $n=n(\lambda, H)$ such that $\lambda_{2}^{n} \leqq 1 / H$. Since $X$ is $\left(\lambda_{1}^{n}, \lambda_{2}^{n}\right)$-HD, there is a finite sequence $b=x_{0}, \ldots, x_{s}$ of points in $X$ such that

$$
\lambda_{1}^{n}\left|x_{i}-x\right| \leqq\left|x_{i+1}-x\right| \leqq \lambda_{2}^{n}\left|x_{i}-x\right|, \quad \lambda_{1}^{n}\left|x_{s}-x\right| \leqq|a-x| \leqq\left|x_{s}-x\right| .
$$

Then $\left|x_{i+1}-x\right| \leqq\left|x_{i}-x\right| / H$ for all $i=0, \ldots, s-1$, and hence $\left|f\left(x_{i+1}\right)-f(x)\right| \leqq$ $\left|f\left(x_{i}\right)-f(x)\right| / h$, which implies $\left|f\left(x_{s}\right)-f(x)\right| \leqq|f(b)-f(x)| / h^{s}$. Furthermore, $|f(a)-f(x)| \leqq H\left|f\left(x_{s}\right)-f(x)\right|$ and thus $\varrho^{\prime} \leqq H h^{-s}$. On the other hand, $|a-x| \geqq$ $\lambda_{1}^{n(s+1)}|b-x|$, which gives $\varrho \geqq \lambda_{1}^{n(s+1)} \geqq h^{-n(s+1)}$ and hence $\varrho^{\prime} \leqq H h \varrho^{1 / n} \leqq H^{2} \varrho^{1 / n}$.

3.11. Remark. The proof of 3.10 gives the following more precise results: We can always choose $C=H^{2}$. If $X$ is connected, we can choose $\alpha=\log H / \log h$. Indeed, in Case 2 of the proof, we can then take $\left|x_{i+1}-x\right|=\left|x_{i}-x\right| / H$ and $|a-x| \geqq$ $\left|x_{s}-x\right| / H$.

3.12. Corollary. Suppose that $X$ is $\lambda$-HD and that $f: X \rightarrow Y$ is $\eta$-QS. Then fis $\eta^{\prime}$-QS, $\eta^{\prime}(t)=C \max \left(t^{\alpha}, t^{1 / \alpha}\right)$, where $C \geqq 1$ and $\alpha \geqq 1$ depend only on $\eta$ and $\lambda$. 
Proof. The conditions of 3.10 are satisfied with $h=1 / \lambda_{1}$ and $H=\max \left(\eta\left(\lambda_{1}^{-1}\right), \eta^{-1}\left(\lambda_{1}\right)^{-1}, \lambda_{1}^{-1}\right)$.

3.13. Remark. If $X$ is connected, we may choose $\lambda_{1}=1 / 2$. Hence we may take in $3.12 C=H^{2}$ and $\alpha=\log H / \log 2$ where $H=\max \left(\eta(2), \eta^{-1}(1 / 2)^{-1}, 2\right)$.

3.14. Theorem. Suppose that $X$ is $\lambda$-HD, that $f: X \rightarrow Y$ is $\eta$-QS and that $A \subset X$ is bounded. Then $f \mid A$ satisfies a two-sided Hölder condition

$$
|x-y|^{\alpha} / c \leqq|f(x)-f(y)| \leqq c|x-y|^{1 / \alpha}
$$

for $x, y \in A$, where the constant $\alpha \geqq 1$ depending only on $\eta$ and $\lambda$ is as in 3.12 and $c$ depends only on $\eta, \lambda, d(A)$ and $d(f A)$.

Proof. By 3.12 we may assume that $\eta(t)=C \max \left(t^{\alpha}, t^{1 / \alpha}\right)$. Let $x, y \in A$. Then 3.5 implies

If $|x-y| \leqq d(A) / 2,3.5$ also gives

$$
|f(x)-f(y)| \geqq \frac{1}{2 C} d(f A)\left(\frac{|x-y|}{d(A)}\right)^{\alpha} .
$$

$$
|f(x)-f(y)| \leqq C d(f A)\left(\frac{2|x-y|}{d(A)}\right)^{1 / \alpha} .
$$

Since $|f(x)-f(y)| \leqq d(f A)$, this is also true when $|x-y| \geqq d(A) / 2$.

3.16. Remarks. 1. Let $X$ be the set consisting of 0 and the numbers $e^{-n !}$, $n=2,3, \ldots$. Define $f: X \rightarrow R^{1}$ by $f(x)=-1 / \log x$ for $x>0$ and $f(0)=0$. A straightforward but tedious proof shows that $f$ is QS. However, $f$ does not satisfy a Hölder condition. Hence the HD-condition is essential in 3.14.

2. Let $X$ be a space with $d(X) \leqq e^{-2}$. Then we can define the logarithmic metric $\delta$ of $X$ by

$$
\delta(x, y)=-\frac{1}{\log |x-y|}
$$

for $x \neq y$ and $\delta(x, x)=0$. It is not difficult to show that an embedding $f: X \rightarrow Y$ with $d(X), d(Y) \leqq e^{-2}$ satisfies the condition (3.15) if and only if $f:(X, \delta) \rightarrow(Y, \delta)$ is a Lipschitz embedding, that is, there is a constant $L \geqq 1$ such that

$$
\delta(x, y) / L \leqq \delta(f(x), f(y)) \leqq L \delta(x, y)
$$

for all $x, y \in X$. In particular, this is true whenever $X$ is HD and $f$ is QS. On the other hand, the condition (3.15) does not imply quasisymmetry. For example, the homeomorphism $f: I^{1} \rightarrow I^{1}$ defined by $f(x)=x$ for $x \leqq 0$ and $f(x)=x^{2}$ for $x>0$ satisfies (3.15) but is not QS.

3.17. Hausdorff dimension. The Hausdorff dimension $\operatorname{dim}_{H} A$ of a set $A$ in a metric space $X$ is the infimum of all numbers $\delta \geqq 0$ such that for every $\varepsilon>0$ there is a countable covering $\left\{A_{j}: j \in \mathbf{N}\right\}$ of $A$ with the property that $d\left(A_{j}\right) \leqq \varepsilon$ for all $j$ and that $\sum_{j} d\left(A_{j}\right)^{\delta+\varepsilon} \leqq \varepsilon$. 
If $f$ satisfies (3.15), then $d(B)^{\alpha} / c \leqq d(f B) \leqq c d(B)^{1 / \alpha}$ for every $B \subset X$. Hence 3.14 gives as a corollary:

3.18. Theorem. Let $X$ be $\lambda$-HD. Let $f: X \rightarrow Y$ be $\eta$-QS. If $A \subset X$, then

$$
\operatorname{dim}_{H} A / \alpha \leqq \operatorname{dim}_{H} f A \leqq \alpha \operatorname{dim}_{H} A
$$

where $\alpha$, depending only on $\eta$ and $\lambda$, is as in Theorem 3.12.

3.19. Theorem. If $X$ is $k$-HTB, then $\operatorname{dim}_{H} X \leqq M(k)<\infty$.

Proof. We may assume that $d(X) \leqq 1$ and that im $k \subset \mathbf{N}$. Set $n=k(2)$. Every set $E \subset X$ can be covered with $n$ sets of diameter at most $d(E) / 2$. Hence we can inductively choose coverings $\mathscr{A}_{i}$ of $X$ such that card $\mathscr{A}_{i}=n^{i}$ and such that $d(A) \leqq 2^{-i}$ for all $A \in \mathscr{A}_{i}$. Let $\alpha>\log n / \log 2$. Then $2^{-\alpha} n<1$, and we obtain

$$
\sum_{A \in \mathscr{A}_{i}} d(A)^{\alpha} \leqq\left(2^{-\alpha} n\right)^{i} \rightarrow 0
$$

as $i \rightarrow \infty$. This proves the theorem with $M(k)=\log k(2) / \log 2$.

3.20. Remark (added November 8, 1979). J. Luukkainen pointed out that a metric space is HTB if and only if it is of finite metric dimension in the sense of P. Assouad, C. R. Acad. Sci. Paris 288, 1979, 731-734. From Remark 2, p. 732, of Assouad it follows that every HTB space can be QS embedded into a euclidean space.

3.21. We shall apply the results of this section to give a characterization of QS embeddings $f: R^{p} \rightarrow R^{n}, p \leqq n$, in terms of compact families of embeddings. If $p=n$, the result is well known; the case $p=n=1$ is due to Beurling and Ahlfors [BA, 2.5], the case $p=n \geqq 2$ to Gehring [Ge, Theorem 18].

By 2.16, an embedding $f: R^{p} \rightarrow R^{n}$ is QS if and only if it is weakly QS. Therefore we simply say that $f$ is $H$-QS if it is weakly $H$-QS.

Fix integers $n \geqq p \geqq 1$. For $H \geqq 1$ let $Q_{H}$ be the family of all $H$-QS embeddings $f: R^{p} \rightarrow R^{n}$ such that $f(0)=0$ and $f\left(e_{1}\right)=e_{1}=(1,0, \ldots, 0)$. As in 3.6 , we consider $C\left(R^{p}, R^{n}\right)$ with the compact-open topology, or equivalently, with the topology of uniform convergence on compact sets. By 3.4, $Q_{H}$ is equicontinuous. By 3.6, $\bar{Q}_{H}$ is compact. By 3.7, every element of $\bar{Q}_{H} \backslash Q_{H}$ is an embedding and hence $H$-QS. Thus $Q_{H}=\bar{Q}_{H}$ is compact.

If $f: R^{p} \rightarrow R^{n}$ is an embedding, we let $W_{f}$ denote the family of all maps $g: R^{p} \rightarrow R^{n}$ such that $g(0)=0, g\left(e_{1}\right)=e_{1}$, and $g=\alpha f \beta$ for some similarity maps $\alpha: R^{n} \rightarrow R^{n}$ and $\beta: R^{p} \rightarrow R^{p}$. If $f$ is $H$-QS, every member of $W_{f}$ is $H$-QS. Therefore $W_{f}$ is contained in the compact family $Q_{H}$.

Conversely, assume that $f: R^{p} \rightarrow R^{n}$ is an embedding such that $W_{f}$ is contained in some compact family $F$ of embeddings $R^{p} \rightarrow R^{n}$. We show that $f$ is QS. Since $F$ and $B_{c}(0,1)$ are compact,

$$
\sup \left\{|g(y)|: y \in B_{c}(0,1), g \in F\right\}=H_{F}<\infty .
$$


Let $a, b, x \in R^{p}$ with $0<|x-a| \leqq|x-b|$. Let $\beta$ be a similarity map of $R^{p}$ such that $\beta(0)=x, \beta\left(e_{1}\right)=b$. Then $\left|\beta^{-1}(a)\right| \leqq 1$. Since $f$ is injective, there is a similarity map $\alpha$ of $R^{n}$ such that $\alpha f \beta(0)=0$ and $\alpha f \beta\left(e_{1}\right)=e_{1}$. Then $g=\alpha f \beta \in W_{f} \subset F$, and hence

$$
\frac{|f(a)-f(x)|}{|f(b)-f(x)|}=\left|g\left(\beta^{-1}(a)\right)\right| \leqq H_{F} .
$$

Thus $f$ is $H_{F}-\mathrm{QS}$. We have proved:

3.22. Theorem. Let $1 \leqq p \leqq n$ and let $f: R^{p} \rightarrow R^{n}$ be an embedding. Then $f$ is QS if and only if $W_{f}$ is contained in a compact family of embeddings $R^{p} \rightarrow R^{n}$.

More precisely, for every $H \geqq 1$ there is a compact family $Q_{H}$ of embeddings $R^{p} \rightarrow R^{n}$ such that $W_{f} \subset Q_{H}$ whenever $f$ is $H$-QS. Conversely, for every compact family $F$ of embeddings $R^{p} \rightarrow R^{n}$ there is $H_{F} \geqq 1$ such that $W_{f} \subset F$ implies that $f$ is $H_{F}$-QS.

\section{Quasisymmetric arcs and curves}

4.1. Terminology. A space $Y$ is an arc if it is homeomorphic to $I=[0,1]$; $Y$ is a curve if it is homeomorphic to the circle $S^{1}=\left\{x \in R^{2}:|x|=1\right\}$. If the homeomorphism can be chosen to be QS, $Y$ is called a quasisymmetric arc or curve.

4.2. Characterization of $\mathrm{QS}$ arcs and curves. Since $I$ and $S^{1}$ are HTB and BT, it follows from 2.10 and 2.11 that every QS arc and curve is HTB and BT. In fact, if $X=I$ or $X=S^{1}$ and if $f: X \rightarrow Y$ is a weakly $H$-QS homeomorphism, the proof of 2.11 shows that $Y$ is $2 H$-BT. However, $Y$ need not be HTB; see Example 4.12.

The purpose of this section is to show that conversely, if $Y$ is an arc or a curve which is HTB and BT, $Y$ is quasisymmetric. By an example we show that the BTproperty alone does not suffice: there is an arc which is BT but not HTB.

For arcs and curves in the plane $R^{2}$, this result is well known from the theory of quasiconformal maps. In fact, if $Y \subset R^{2}$ is a BT arc or curve, there is a quasiconformal homeomorphism $f: R^{2} \rightarrow R^{2}$ mapping $Y$ onto $I$ or $S^{1}$. See [LV, II.8.7] and $[\mathrm{Ri}]$.

4.3. Subdivisions. Throughout this section we assume that all arcs and curves are oriented. Subarcs have the induced orientation. The initial point of an arc $J$ is denoted by $A(J)$, the terminal point by $B(J)$. A subdivision of $J$ is a finite sequence of subarcs $J_{1}, \ldots, J_{n}$ such that $J=J_{1} \cup \ldots \cup J_{n}, A(J)=A\left(J_{1}\right), B(J)=B\left(J_{n}\right)$ and that $A\left(J_{i+1}\right)=B\left(J_{i}\right)$ for $i<n$. If $J$ is a curve, $J_{1}, \ldots, J_{n}$ is a subdivision of $J$ if either $J_{1}=J$ or $n>1$ and $J_{i}$ are subarcs such that $A\left(J_{1}\right)=B\left(J_{n}\right)$ and that $J_{1}, \ldots, J_{n-1}$ is a subdivision of $J \backslash$ int $J_{n}$.

4.4. Lemma. Let $k:[1 / 2, \infty) \rightarrow[1, \infty)$ be increasing, $c \geqq 1$ and $0<\varepsilon<1$. Then there are numbers $\delta=\delta(k, c, \varepsilon)>0$ and $p=p(k, c, \varepsilon) \in \mathbf{N}$ such that if $J$ is an arc. 
or a curve, which is $k$-HTB and c-BT, there is a subdivision $J_{1}, \ldots, J_{p}$ of $J$ with

for $i \leqq p$.

$$
\delta d(J) \leqq d\left(J_{i}\right) \leqq \varepsilon d(J)
$$

Proof. We may assume that im $k \subset \mathbf{N}$. We will show that the lemma is true with $p=k(4 c / \varepsilon)$ and $\delta=2^{-p-1} \varepsilon$. By a change of scale we may assume that $d(J)=1$.

We define inductively arcs $J_{1}, \ldots, J_{n+1}$ as follows: Let $a=A(J)$, if $J$ is an arc; if $J$ is a curve, let $a$ be an arbitrary point of $J$. Let $J_{1}$ be the maximal subarc of $J$ such that $A\left(J_{1}\right)=a$ and such that $J_{1} \subset B_{c}(a, \varepsilon / 4)$. Since $\varepsilon<1$, there is such an arc $J_{1}$, and $\varepsilon / 4 \leqq d\left(J_{1}\right) \leqq \varepsilon / 2$. Suppose that $J_{1}, \ldots, J_{i}$ have been constructed. If $J_{1} \cup \ldots \cup J_{i}=J$, the process ends. Otherwise we let $J_{i+1}$ be the maximal subarc of $J \backslash$ int $\left(J_{1} \cup \ldots \cup J_{i}\right)$ such that $A\left(J_{i+1}\right)=B\left(J_{i}\right)$ and such that $J_{i+1} \subset B_{c}\left(B\left(J_{i}\right), \varepsilon / 4\right)$. Since $J$ is compact, the process ends after a finite number of steps, and we obtain a subdivision $J_{1}, \ldots, J_{n+1}$ of $J$ satisfying $d\left(J_{i}\right) \leqq \varepsilon / 2$ for all $i$ and $d\left(J_{i}\right) \geqq \varepsilon / 4$ for $i \leqq n$. Deleting $J_{n+1}$ and replacing $J_{n}$ by the subarc with the initial point $A\left(J_{n}\right)$ and the terminal point $B(J)$ (if $J$ is an arc) or $a$ (if $J$ is a curve), we get a subdivision $J_{1}, \ldots, J_{n}$ of $J$ such that $\varepsilon / 4 \leqq d\left(J_{i}\right) \leqq \varepsilon$ for all $i$.

Assume that $1 \leqq i<j \leqq n$ and let $J^{\prime}$ be a subarc of $J$ with endpoints $A\left(J_{i}\right)$ and $A\left(J_{j}\right)$. Then $d\left(J^{\prime}\right) \geqq \varepsilon / 4$, which implies $\left|A\left(J_{i}\right)-A\left(J_{j}\right)\right| \geqq \varepsilon / 4 c$. Since $J$ is $k$-HTB, this yields $n \leqq k(4 c / \varepsilon)=p$. If $n=p$, there is nothing more to be done. Otherwise we divide $J_{1}$ into two subarcs of diameter at least $\varepsilon / 8$. Repeating this $p-n$ times we obtain a new subdivision written as $J_{1}, \ldots, J_{p}$, such that $2^{-p-1} \varepsilon \leqq d\left(J_{i}\right) \leqq \varepsilon$ for all $i \leqq p$.

4.5. Some constructions. Let $k$ and $c$ be as in Lemma 4.4. We fix $\varepsilon=1 / 2$ and obtain by 4.4 numbers $\delta=\delta(k, c, 1 / 2)=\delta(k, c)$ and $p=p(k, c, 1 / 2)=p(k, c)$. We may assume that $\delta \leqq 1 / 4$. We set $\varrho=\varrho(k, c)=\varepsilon / \delta=1 / 2 \delta$. Then $\varrho \geqq 2$ and $\varrho^{2} \geqq 1 / \delta$.

We next choose positive integers $m=m(k, c) \geqq 2$ and $n=n(k, c)$ such that

$$
2^{n} \geqq \varrho^{m},
$$

$$
4 n+3 \leqq p^{m} \text {. }
$$

To see the existence of such $m$ and $n$, find first $m$ and $n$ that satisfy the first inequality. If they do not satisfy the second inequality, double them, and repeat this until the second inequality is also satisfied.

We set $N=N(k, c)=p^{m}+2 n(p-1)$.

Let $W$ be the set of words that can be formed of the numbers $1, \ldots, N$. Thus the elements of $W$ are finite sequences $w=n_{1} \ldots n_{q}$ where $1 \leqq n_{i} \leqq N$ and $q \geqq 0$; also the empty sequence $\emptyset$ is in $W$. The length $l(w)$ of a word $w=n_{1} \ldots n_{q}$ is the number $q \geqq 0$. Two words $w=n_{1} \ldots n_{q}$ and $v=m_{1} \ldots m_{r}$ can be concatenated to a new word $w v=n_{1} \ldots n_{q} m_{1} \ldots m_{r}$.

4.7. Lemma. Let $J$ be an arc or a curve, which is $k$-HTB and c-BT. Then there are subarcs $J_{w} \subset J, w \in W, \quad\left(J_{\emptyset}\right.$ is a curve if $J$ is a curve) such that: 
(i) $J_{\emptyset}=J$.

(ii) $J_{w 11}, \ldots, J_{w N}$ is a subdivision of $J_{w}$ for $w \in W$.

(iii) $d\left(J_{w}\right) \leqq 2^{-l(w)} d(J)$.

(iv) If $v, w \in W, l(v)=l(w)$, and if $J_{v}$ and $J_{w}$ are adjacent, then

$$
\varrho^{-m-2} \leqq d\left(J_{v}\right) / d\left(J_{w}\right) \leqq \varrho^{m+2} .
$$

Proof. We construct the $\operatorname{arcs} J_{w}$ by induction on $l(w)$. We set $J_{\emptyset}=J$. Assume then that we have constructed $J_{w}$ if $l(w)<s$ such that the conditions of 4.7 are satisfied. We construct now the arcs $J_{w}, l(w)=s$.

We first divide every $J_{w}, l(w)=s-1$, into $p$ subarcs as in Lemma 4.4. Each of these subarcs is again divided into $p$ subarcs as in Lemma 4.4. We repeat this $m$ times and thus divide $J_{w}$ into $M=p^{m}$ subarcs, denoted $J_{w 1}^{\prime}, \ldots, J_{w M}^{\prime}$. These satisfy

$$
\begin{gathered}
\delta^{m} d\left(J_{w}\right) \leqq d\left(J_{w i}^{\prime}\right) \leqq 2^{-m} d\left(J_{w}\right), \\
\varrho^{-m} \leqq d\left(J_{w i}^{\prime}\right) / d\left(J_{w j}^{\prime}\right) \leqq \varrho^{m}
\end{gathered}
$$

for all $i \leqq M$ and $j \leqq M$.

We now fix $w \in W, l(w)=s-1$. By (4.8), the ratios $d\left(J_{w i}^{\prime}\right) / d\left(J_{w(i+1)}^{\prime}\right)$ lie in the desired range, but this may not be true for $r=d\left(J_{w 1}^{\prime}\right) / d\left(J_{v M}^{\prime}\right)$ when $J_{v M}^{\prime}$ and $J_{w 1}^{\prime}$ are adjacent, $l(v)=s-1$. However, in any case we have $\varrho^{-2 m-2} \leqq r \leqq \varrho^{2 m+2}$. This follows from (4.8) and from the inductive hypothesis. If $r \in\left[\varrho^{-m-2}, \varrho^{m+2}\right]$, we do nothing. If this is not true, we must perform some further subdivision. Assume, for instance, that $r>\varrho^{m+2}$. Then we subdivide $J_{w 1}^{\prime}$ into $p$ subarcs $J_{w 11}^{\prime}, \ldots, J_{w 1 p}^{\prime}$ as in Lemma 4.4. If still $d\left(J_{w 11}^{\prime}\right) / d\left(J_{v M}^{\prime}\right)>\varrho^{m+2}$, we repeat this with respect to $J_{w 11}^{\prime}$. We continue in this way and stop as soon as we get $J_{w 1 \ldots 1}^{\prime}=J_{u}^{\prime}$ such that $d\left(J_{u}^{\prime}\right) / d\left(J_{v M}^{\prime}\right) \leqq$ $\varrho^{m+2}$. By (4.6), this happens after at most $n$ steps. Since $\delta \geqq \varrho^{-2}$, we have $d\left(J_{u}^{\prime}\right) / d\left(J_{v M}^{\prime}\right) \geqq$ $\varrho^{m}>\varrho^{-m-2}$. If $r<\varrho^{-m-2}$, we subdivide $J_{v M}^{\prime}$ instead. We do this for all $w \in W$, $l(w)=s-1$. We get new subdivisions $J_{w i}^{\prime \prime}, 1 \leqq i \leqq N_{w}$, of the $\operatorname{arcs} J_{w}$. Here $M \leqq N_{w} \leqq$ $M+2 n(p-1)=N$ and $N-N_{w}$ is divisible by $p-1$.

If $N_{w}<N$, we must still perform some subdivision. Let $d_{w}=\left(N-N_{w}\right) /(p-1)$. We subdivide the arcs $J_{w 3}^{\prime}, J_{w 5}^{\prime}, \ldots, J_{w, 1+2 d_{w}}^{\prime}$, which are also arcs in the subdivision $J_{w 1}^{\prime \prime}, \ldots, J_{w N_{w}}^{\prime \prime}$, into $p$ subarcs as in Lemma 4.4. We get a new subdivision $J_{w 1}, \ldots, J_{w N}$ of $J_{w}$. This is the final subdivision. Note that the arcs $J_{w 1}^{\prime}, J_{w 3}^{\prime}, \ldots, J_{w, 1+2 d_{w}}^{\prime}, J_{w M}^{\prime}$ exist and are pairwise disjoint, since $d_{w} \leqq 2 n$ and $4 n+3 \leqq M$.

We must show that the arcs $J_{w}, w \in W, l(w) \leqq s$, satisfy (i)-(iv). The conditions (i) and (ii) are trivial, and (iii) follows from (4.8). We prove (iv). If $J_{v N}$ and $J_{w 1}$ are adjacent, $v, w \in W, l(v)=l(w)=s-1$, then $d\left(J_{w 1}\right) / d\left(J_{v N}\right) \in\left[\varrho^{-m-2}, \varrho^{m+2}\right]$ by construction. If $J_{w i}$ and $J_{w j}$ are adjacent and both of them are of the form $J_{w q}^{\prime}$, then $r=d\left(J_{w i}\right) / d\left(J_{w j}\right) \in\left[\varrho^{-m}, \varrho^{m}\right]$. If only one of them is of the form $J_{w q}^{\prime}$, then $r \in\left[\varrho^{-m} \delta, \varrho^{m} / \delta\right] \subset\left[\varrho^{-m-2}, \varrho^{m+2}\right]$. If neither of them is of this form, $r \in[\delta / \varrho, \varrho / \delta] \subset$ $\left[\varrho^{-m-2}, \varrho^{m+2}\right]$.

We are now ready to prove the main theorem of this section. 
4.9. Theorem. Let $J$ be an arc or a curve. Then $J$ is QS if and only if $J$ is HTB and $\mathrm{BT}$.

More precisely, if $J$ is $k$-HTB and c-BT, there is an $\eta$-QS homeomorphism $f: X \rightarrow J$, $X=I$ or $S^{\mathbf{1}}$, with $\eta$ depending only on $k$ and $c$. Conversely, if $J=f X$ for some $\eta-\mathrm{QS}$ homeomorphism $f$, then $J$ is $k$-HTB and $c$-BT with $k$ and $c$ depending only on $\eta$.

Proof. In 4.2 we already proved the last statement of the theorem with $c=2 \eta(1)$. Inspection of the proof of 2.10 would give the explicit estimate $k(\alpha)=1+2 \pi / \eta^{-1}(1 / 2 \alpha)$.

Conversely, assume that $J$ is $k$-HTB and $c$-BT. In view of 2.15 , it suffices to find a homeomorphism $f: X \rightarrow J$ which is weakly $H$-QS with $H=H(k, c)$.

If $X=S^{1}$, we let $|a-b|$ be $1 / 2 \pi$ times the length of the shorter arc joining the points $a$ and $b$ in $S^{1}$. Then $|a-x| \leqq|b-x|$ if and only if $\|a-x\| \leqq\|b-x\|$ where $\|y\|$ is the ordinary inner product norm of $y \in R^{2}$. Consequently, both metrics give the same class of weakly $H$-QS embeddings of $S^{\mathbf{1}}$.

Let $N=N(k, c)$ be as in 4.5, and choose the subarcs $J_{w}, w \in W$, of $J$ as in 4.7. We subdivide $X$ into $N$ subarcs $I_{1}, \ldots, I_{N}$ of length $1 / N$. These in turn are divided into $N^{2}$ subarcs $I_{11}, \ldots, I_{N N}$. Continuing in this manner we get subarcs $I_{w}$ for $w \in W$ like the subarcs $J_{w}$ of $J$. The length of $I_{w}$ is $N^{-l(w)}$. There is a unique homeomorphism $f: X \rightarrow J$ such that $f I_{w}=J_{w}$ for all $w \in W$. We will show that $f$ is weakly $H(k, c)-\mathrm{QS}$.

Let $a, b, x \in X$ with $0<|a-x| \leqq|b-x|$. Let $s$ be the smallest integer such that $N^{-s} \leqq|b-x| / 2$. Let $I^{\prime}$ be the shortest arc of $X$ with endpoints $b$ and $x$. Then $I^{\prime}$ contains an $\operatorname{arc} I_{w}$ with $l(w)=s$. Then

$$
|f(b)-f(x)| \geqq d\left(f I^{\prime}\right) / c \geqq d\left(J_{w}\right) / c .
$$

On the other hand, $N^{-s+1} \geqq|b-x| / 2 \geqq|a-x| / 2$, implying $|a-x| \leqq 2 N^{-s+1}$ and $|a-b| \leqq|a-x|+|x-b| \leqq 4 N^{-s+1}$. Hence there is a sequence of arcs $I_{w(1)}, \ldots, I_{w(r)}$ following one another such that $w(i) \in W, l(w(i))=s, r \leqq 4 N+1, w(1)=w$, and $a \in I_{w(r)}$. In the same manner, $|b-x| \leqq 2 N^{-s+1}$ implies that there is a sequence of arcs $I_{v(1)}, \ldots, I_{v\left(r^{\prime}\right)}$ following one another such that $v(i) \in W, l(v(i))=s, r^{\prime} \leqq$ $2 N+1, v(1)=w$ and $x \in I_{v\left(r^{\prime}\right)}$. Let $M=\varrho^{m+2}$ where $\varrho$ is as in 4.5. Then (iv) of Lemma 4.7 implies that for every $u \in I_{w}$, we have $|f(a)-f(u)| \leqq\left(1+M+\ldots+M^{r}\right) d\left(J_{w}\right) \leqq$ $5 N M^{5 N} d\left(J_{w}\right)$ and $|f(x)-f(u)| \leqq\left(1+M+\ldots+M^{r^{\prime}}\right) d\left(J_{w}\right) \leqq 3 N M^{3 N} d\left(J_{w}\right)$. Thus $|f(a)-f(x)| \leqq 8 N M^{5 N} d\left(J_{w}\right)$. Together with (4.10) this gives $|f(a)-f(x)| \leqq$ $8 c N M^{5 N}|f(b)-f(x)|$. Hence $f$ is weakly $H$-QS with $H=8 c N M^{5 N}=H(k, c)$.

4.11. Corollary. An arc or a curve in $R^{n}$ is QS if and only if it is BT.

4.12. Example. In 2.8 we constructed an arc which is HTB but not BT. We will now construct an arc which is BT but not HTB, and hence not QS. Let $R^{\infty}$ be the space of all sequences $x=\left(x_{1}, x_{2}, \ldots\right)$ such that $x_{j} \neq 0$ for only finitely many $j$. Let the metric of $R^{\infty}$ be induced by the norm $|x|=\max _{j}\left|x_{j}\right|$. Let $e_{1}, e_{2}, \ldots$ be the standard basis of $R^{\infty}$ and let $u_{j}=e_{j} / j$. Let $\Delta_{j}$ be the line segment joining $u_{j}$ 
and $u_{j+1}$. Then the union $A$ of all $\Delta_{j}$ and the origin is an arc, and it is easily seen to be BT. However, $A$ is not HTB. To see this, consider the ball $B=B_{c}(0,1 / n) \cap A$. Then $u_{j} \in B$ for $j \geqq n$. If $n \leqq i<j \leqq 2 n$, then $\left|u_{i}-u_{j}\right|=1 / i \geqq 1 / 2 n$. Thus $B$ contains $n+1$ points of mutual distance $\geqq 1 / 2 n$. If $A$ were $k$-HTB, this would imply $n+1 \leqq$ $k$ (2) for all $n$ and hence a contradiction.

We remark that a homeomorphism $f:[0,1] \rightarrow A$, which is weakly QS, is obtained as follows: $f(0)=0, f\left(2^{-j}\right)=u_{j+1}$, and $f$ maps $\left[2^{-j-1}, 2^{-j}\right]$ affinely onto $\Delta_{j+1}$. The inverse map $f^{-1}$ is not weakly QS.

\section{References}

BA] Beurling, A., and L. Ahlfors: The boundary correspondence under quasiconformal mappings. - Acta Math. 96, 1956, 125-142.

[Du] Dugundi, J.: Topology. - Allyn and Bacon, Inc., Boston, 1966.

[Ge] Gehring, F.W.: Rings and quasiconformal mappings in space. - Trans. Amer. Math. Soc. 103, 1962, 353-393.

[Ks] Kelingos, J. A.: Boundary correspondence under quasiconformal mappings. - Michigan Math. J. 13, 1966, 235-249.

[Ky] Kelley, J. L.: General topology. - D. Van Nostrand Company, Inc., Toronto-New YorkLondon, 1955.

[LV] Lehto, O., and K. I. Virtanen: Quasiconformal mappings in the plane. - Springer-Verlag. Berlin-Heidelberg-New York, 1973.

[Re $\left.{ }_{1}\right]$ RengGli, H.: Doppelverhältnisse und quasikonforme Abbildungen. - Comment. Math. Helv, 43, 1968, 161-175.

[Re $\left.\mathrm{Re}_{2}\right]$ ReNGGLI, H.: On triangular dilatation. - Proceedings of the Romanian-Finnish seminar on Teichmüller spaces and quasiconformal mappings, Braşov, 1969, edited by Cabiria Andreian Cazacu, Publishing House of the Academy of the Socialist Republic of Romania, 1971, 255-259.

[Ri] Rickman, S.: Characterization of quasiconformal arcs. - Ann. Acad. Sci. Fenn. Ser. A I 395, 1966, 1-30.

[V $\ddot{1}_{1}$ ] VÄıs̈̈LÄ, J.: Lectures on $n$-dimensional quasiconformal mappings. - Lecture Notes in Mathematics 229, Springer-Verlag, Berlin-Heidelberg-New York, 1971.

[Vä] VÄıs̈̈LÄ, J.: Quasisymmetric embeddings in euclidean spaces. - Trans. Amer. Math. Soc. (to appear).

University of Helsinki

Department of Mathematics

SF-00100 Helsinki 10

Finland

Received 27 September 1979 